\title{
SOME REMARKS ON ORDER TYPES AND DECOMPOSITIONS OF SETS $\left({ }^{(}\right)$
}

\author{
BY \\ SEYMOUR GINSBURG
}

This paper is a sequel to the author's previous article on order types [3], to which the reader is referred for all unfamiliar terms and definitions. The two principal problems under investigation here are:

(P) to study the existence of order types $\tau$ such that $\sigma<\tau<\bar{E}$, where $E$ and $\sigma, \sigma$ being an order type $<\bar{E}$, are given; and

$(Q)$ to decompose a set into the union of disjoint sets which have some special properties.

$\$ 1$ deals with conditions on $E$ and $\sigma$ which guarantee the existence of at least one order type $\tau$ in problem (P). In particular, considerable attention is focused on the case where $\bar{E} \equiv \alpha \omega$. In $\S 2$ conditions are stated which ensure the existence of precisely $n$ distinct order types $\tau$, where $n$ is any nonnegative integer. Finally, some scattered results pertaining to problem $(Q)$ are given (\$3). For example: (1) each linear set of power $2^{\mathrm{N}_{0}}$ is the union of $2^{\text {so }_{0}}$ disjoint, exact sets (Theorem 3.1); and (2) if $A$ and $B$ are any two disjoint, similar sets whose union is the set of real numbers, then to each point $p$ of $A$ there corresponds a similarity transformation $f_{p}$ of $A$ into itself for which $f_{p}(p) \neq p$ (Theorem 3.2).

1. Sufficiency conditions for problem (P). As in [3], the set of real numbers is designated by " $R$. " " $\lambda$ " will represent the order type of the real numbers, and " $\eta$ " the rationals ordered in the natural manner. " $\theta$ " will designate the smallest ordinal number whose power is $2^{\mathrm{N}_{0}}$.

LEMma 1.1. Let $E$ be any linear set and $A$ any subset of $E$. If $f$ is a similarity transformation of $A$ into $R$, then $f$ can be extended to become a similarity transformation $g$ of a Borel set with respect to $E, B$, into $R$.

Proof. By Lemma 2 of [3], $f$ can be extended to become a similarity transformation $h$ of a Borel set $H$ into $R$. Let $B=H \cap E$ and $g(x)=h(x)$ for $x$ in $B$. The set $B$ and the function $g$ then satisfy the conclusion of the lemma.

Notation. By $\bar{A} \leqq \bar{B}$ is meant that either $\bar{A} \equiv \bar{B}$ or $\bar{A}<\bar{B}$.

THEOREM 1.1. Let $E$ be a linear set of power $2^{\text {No. }}$. Let $\sigma$ be an order type $<\bar{E}$ which has the property that if $\bar{A} \leqq \sigma$, then the power of the set $E-A$ is $2^{* 0}$. Then there exists an order type $\tau$ for which $\sigma<\tau<\bar{E}$.

Received by the editors April 24, 1952.

(1) $\$ \$ 1$ and 2 of this paper are drawn from my $\mathrm{Ph} . \mathrm{D}$. thesis, submitted to the University of Michigan, August 1952. 
Proof. Denote by $B$ a subset of $E$ for which $\bar{B}=\sigma$.

CASE (a). Suppose that the power of $B$ is $2^{\text {No }}$. Let $H=\{D\}$ be the family of those Borel sets with respect to $B$ which are of power $2^{N_{0}}$ each. For each set $D$ there are $2^{N_{0}}$ similarity transformations of $D$ into $R$. Let $G=\{g\}$ be the family of similarity transformations of $E$ into itself. The power of $G$ is at most $2^{N_{0}}$. Therefore the power of the family $S=\{s=(D, f, g) \mid D \in H, f$ a similarity transformation of $D$ into $E, g \in G\}$ is $2^{N_{0}}$. Well order the elements of $R$ and $S$ into the two sequences $\left\{x_{\xi}\right\}, \xi<\theta$, and $\left\{s_{\xi}\right\}, \xi<\theta$, respectively, where $s_{\xi}=\left(D_{\xi}, f_{\xi}, g_{\xi}\right)$. By transfinite induction we shall define three sequences of elements of $R,\left\{p_{\xi}\right\}, \xi<\theta,\left\{q_{\xi}\right\}, \xi<\theta$, and $\left\{r_{\xi}\right\}, \xi<\theta$.

Let $\gamma$ be any ordinal number which is smaller than $\theta$. Since $\bar{D}_{\gamma} \leqq \bar{B}<\bar{E}$, it follows that

$$
\overline{g_{\gamma}^{*}\left[f_{\gamma}\left(D_{\gamma}\right)\right]} \leqq \bar{B}
$$

where $g_{\gamma}^{*}$ is the inverse function of $g_{\gamma}$. Therefore the power of the set $E$ $-g_{\gamma}^{*}\left[f_{\gamma}\left(D_{\gamma}\right)\right]$ is $2^{\mathrm{No}}$. Since the power of the set $g_{\gamma}(E)-f_{\gamma}\left(D_{\gamma}\right)$ is equal to the power of the set $E-g_{\gamma}^{*}\left[f_{\gamma}\left(D_{\gamma}\right)\right]$, it follows that the power of $g_{\gamma}(E)-f_{\gamma}\left(D_{\gamma}\right)$ is $2^{N_{0}}$. We conclude that if $C$ is a set of power $<2^{N_{0}}$, then the power of $g_{\gamma}(E)-\left[f_{\gamma}\left(D_{\gamma}\right) \cup C\right]$ is $2^{\aleph_{0}}$. Denote by $p_{0}$ and $q_{0}$ the first two elements of $g_{0}(E)$ $-f_{0}\left(D_{0}\right)$, and by $r_{0}$ the first element of $f_{0}\left(D_{0}\right)-\left\{p_{0}, q_{0}\right\}$. Now suppose that the elements $p_{\xi}, q_{\xi}$, and $r_{\xi}$ have been defined for each $\xi<\mu$ where $\mu<\theta$. Let $p_{\mu}$ and $q_{\mu}$ be the first two elements of the set

$$
g_{\mu}(E)-\left[f_{\mu}\left(D_{\mu}\right) \cup\left\{p_{\xi}, q_{\xi}, r_{\xi} / \xi<\mu\right\}\right] .
$$

Denote by $r_{\mu}$ the first element of the set

$$
f_{\mu}\left(D_{\mu}\right)-\left[\left\{p_{\xi}, q_{\xi} / \xi \leqq \mu\right\} \cup\left\{r_{\xi} / \xi<\mu\right\}\right] .
$$

Since the power of the set $f_{\mu}\left(D_{\mu}\right)$ is $2^{N_{0}}$, the element $r_{\mu}$ exists. Let $M=\left\{p_{\xi} \mid \xi<\theta\right\} \cap E$ and $N=B \cup M$. Clearly the power of $M$ is $2^{N_{0}}$. We shall show that $\bar{B}<\bar{N}<\bar{E}$.

To see this we shall first demonstrate that $\bar{M}$ and $\bar{B}$ are incomparable ${ }^{2}$ ). Suppose that $f$ is a similarity transformation of $B$ into $M$. As $B$ is a Borel set with respect to $B$, for some ordinal number $\delta, s_{\delta}=(B, f, I)$, where $I$ is the identity transformation of $E$. The element $r_{\delta}$ is an element of the set $f(B)-M$. Thus it is impossible to have $f(B) \subseteq M$. Now suppose that $k$ is a similarity transformation of $M$ into $B$, i.e. $k(M) \subseteq B$. Denote by $m$ the function defined by $m[k(x)]=x$ for $x$ in $M$. By Lemma 1.1 , the function $m$ may be extended to become a similarity transformation $f$ of a Borel set with respect to $B, Q$, where $k(M) \subseteq Q$. Hence, for some $\delta$, we have $s_{\delta}=(Q, f, I)$. It follows that $p_{\delta}$ is in the set $E-f(Q)$. As $E-f(Q) \subseteq E-M, p_{\delta}$ is in $E-M$. Since $p_{\delta}$ is also in

(2) This result is stronger than that which is actually needed to demonstrate $\bar{B}<\bar{N}<\bar{E}$. We shall use only the fact that $\bar{M} \leqq \bar{B}$ is false. 
$M$, we again are led to a contradiction. Thus $\bar{M}$ and $\bar{B}$ must be incomparable. Consider the sets $N, B$, and $E$. Clearly $\bar{B} \leqq \bar{N} \leqq \bar{E}$. From the fact that $\bar{M} \leqq \bar{B}$ is false, it follows that there is no similarity transformation of $N$ into $B$. Thus $\bar{B}<\bar{N}$. Now suppose that $g$ is a similarity transformation of $E$ into $N$. For some $\delta$, we have $s_{\delta}=(B, \bar{f}, g)$, where $\bar{f}$ is the identity transformation of $B$. The element $q_{\delta}$ is in $g(E)-B$ and is not in $M$. Hence $q_{\delta}$ is an element of $g(E)-(B \cup M)$, i.e., $q_{\delta}$ is in $g(E)-N$. Consequently it is impossible to have $g(E) \subseteq N$. We conclude that $\bar{N}<\bar{E}$.

CASE (b). Suppose that the power of $B$ is $<2^{N_{0}}$. We modify the argument in case (a) slightly, by defining $p_{\xi}$ and $q_{\xi}$ only for $\xi<\theta$. A similar line of reasoning then shows that $\bar{N}<\bar{E}$. Since the power of $B$ is smaller than the power of $N$, it follows that $\bar{B}<\bar{N}$. Q.E.D.

By the use of Lemma 1.1 it is readily seen that the assumptions of the following corollary imply the assumptions of Theorem 1.1, whence its conclusion.

Corollary 1.1. Let $E$ be a linear set of power $2^{\mathrm{N}_{0}}$. Let $A$ be a subset of $E$ which has the property that if $B$ is any Borel subset of $A$, with respect to $A$, and $f$ is any similarity transformation of $B$ into $R$, then the power of $E-f(B)$ is $2^{\mathrm{No}}$. Then there exists an order type $\tau$ for which $\bar{A}<\tau<\bar{E}$.

CoROLlary 1.2. Let $E$ be a linear set of power $2^{\mathbb{N}_{0}}$, and have the property that if $A \subset E$ and $\bar{A}<\bar{E}$, then the power of $E-A$ is $2^{\mathrm{N}_{0}}$. Then for each order type $\sigma<\bar{E}$, an order type $\tau$ can be found for which $\sigma<\tau<\bar{E}$.

It is natural to inquire as to when the hypotheses of Corollary 1.2 are satisfied. Sierpiński has shown that they are when $\bar{E} \equiv \lambda[6$, p. 261]. In order to set up a sufficient condition for the hypothesis of Corollary 1.2 to be satisfied, by generalizing Sierpiński's proof, we introduce

Definition. A nonempty linear set $E$ is said to have a "similarity decomposition" if $E$ is the union of $2^{\aleph_{0}}$ disjoint sets $E_{\xi}$, where $\bar{E}_{\xi} \equiv \bar{E}$. The order type $\sigma$ is said to have a similarity decomposition if $\sigma=\bar{E}$, where $E$ has a similarity decomposition.

Lemma 1.2. Let $E$ have a similarity decomposition. If $A \subset E$ and $\bar{A}<\bar{E}$, then the power of the set $E-A$ is $2{ }^{\mathrm{No}}$.

Proof. Let $E$ be the union of $2^{\aleph_{0}}$ disjoint sets $E_{\xi}$, where $\bar{E}_{\xi} \equiv \bar{E}$. Since $\bar{A}<\bar{E} \equiv \bar{E}_{\xi}$, it follows that $E_{\xi}-A \neq \phi$. Thus the power of $E-A$ is $2^{\text {Noo. }}$.

Corollary 1.3. If $E$ has a similarity decomposition, then for each order type $\sigma<\bar{E}$, an order type $\tau$ can be found for which $\sigma<\tau<\bar{E}$.

As yet, I know of no order type, distinct from $\lambda$, which has a similarity decomposition.

The reasoning in Theorem 1.1 also shows 
THEOREM 1.2. Let $E$ be a linear set of power $2^{\aleph_{0}}$. Let $\sigma$ be an order type $<\bar{E}$, of power $2^{N_{0}}$, which has the property that if $\bar{A} \leqq \sigma$, then the power of the set $E-A$ is $2^{\aleph_{0}}$. Then there exists an order type $\tau<\bar{E}$ of power $2^{\aleph_{0}}$, such that $\tau$ is incomparable with $\sigma$.

Trivially there exist corollaries to Theorem 1.2 which parallel the corollaries following Theorem 1.1 .

The power condition on $\sigma$ is needed. Consider the order types $\sigma=n$, where $n$ is any positive integer, and $\sigma=\eta$. If $\sigma=n$, then $\sigma$ is comparable with every order type. If $\sigma=\eta$ and $\tau \leqq \bar{E}$, where $\tau$ is any order type with a nondenumerable power, then, as is well known, $\sigma<\tau$, i.e., every nondenumerable linear set contains a subset similar to the rational numbers. If $\sigma=\eta$ and $\tau \leqq \bar{E}$, where $\tau$ is any order type with a denumerable power, then $\tau \leqq \eta$. In either case $\sigma$ is comparable with $\tau$.

The remainder of this section shall be concerned with the existence of $\tau$ when $E$ has the special property that $\bar{E} \equiv \alpha \omega$, where $\alpha$ is any order type. In preparation for Theorem 1.3 we prove

LEMMA 1.3. If $\alpha$ is any order type for which $\alpha<\alpha 2$, then $\alpha n<\alpha(n+1)$ for any positive integer $n$.

Proof. By hypothesis, the conclusion of the lemma is true for $n=1$. Using induction suppose that $\alpha n<(n+1)$ for $n=1, \cdots, k-1$. Let $\left\{A_{i}\right\}, 0<i<\omega$, be a sequence of disjoint sets in which each $\bar{A}_{i}=\alpha$, and denote by $B$ the set $B=\mathrm{U}_{0<n<\omega} A_{n}$. Order the elements of $B$ in the following manner. If $x_{n}$ and $y_{n}$ are each elements of $A_{n}$ and $x_{n}<y_{n}$ in $A_{n}$, let $x_{n}<y_{n}$ in $B$. If $x_{n}$ is in $A_{n}$ and $x_{m}$ is in $A_{m}$, where $n<m$, let $x_{n}<x_{m}$. For each $n$, let $B_{n}=\bigcup_{0<i \leqq n} A_{i}$. Now suppose that $\alpha k<\alpha(k+1)$ is false, i.e., there exists a similarity transformation $f$ which maps $B_{k+1}$ into $B_{k}$. For any element $\bar{x}$ in $A_{k+1}$, consider the point $f(\bar{x})$. If $f(\bar{x})$ were to be an element of $A_{j}$, where $j<k$, then $f(y)<f(\bar{x})$ for $y$ in $B_{k}$. Thus $f\left(B_{k}\right)$ would be a subset of $B_{k-1}$. This in turn would contradict our induction hypothesis that $\alpha(k-1)<\alpha k$. Therefore $f(\bar{x})$ must be an element of $A_{k}$. Similar reasoning shows that the element $f[f(\bar{x})]$ is also in $A_{k}$. Consequently

$$
f\left(A_{k+1}\right) \cup f\left[f\left(A_{k+1}\right)\right] \subseteq A_{k},
$$

and as

$$
f\left(A_{k+1}\right) \cap f\left[f\left(A_{k+1}\right)\right]=\phi,
$$

we conclude that $\alpha 2 \leqq \alpha$. Since this contradicts our assumption that $\alpha<\alpha 2$, no such similarity transformation $f$ can exist. Thus $\alpha k<(k+1)$.

By induction the lemma then holds for all positive integers $n$.

REMARK. The above proof may be generalized to show that if $\alpha$ is any order type for which $\alpha<\alpha 2$, then $\alpha \gamma<\alpha(\gamma+1)$ for each nonzero ordinal number $\gamma$. 
TheOREM 1.3. Let $\alpha$ be any order type for which $\alpha<\alpha 2$. If $\sigma$ is any order type for which $\sigma<\alpha \omega$, then there exists an order type $\tau$ such that $\sigma<\tau<\alpha \omega$.

Proof. Let $A_{i}, B_{j}$, and $B$ be the same as in the preceding lemma. Hence $\bar{B}$ $=\alpha \omega$ and $\alpha<\alpha 2<\alpha 3<\ldots$. Let $D$ be a subset of $B$ for which $\bar{D}=\sigma$, and $D_{n}=B_{n} \cap D$. If $\sigma \leqq \alpha n_{0}$ for some positive integer $n_{0}$, then for $\tau=\alpha\left(n_{0}+1\right)$,

$$
\sigma \leqq \alpha n_{0}<\alpha\left(n_{0}+1\right)<\alpha \omega .
$$

Therefore we shall suppose that $\sigma \leqq \alpha n$ is false for all $n$. For each $n$ therefore, either $\alpha n<\sigma$, or else $\alpha n$ is incomparable with $\sigma$. Suppose the former possibility occurs for all $n$, i.e., $\alpha n<\sigma$ for $n=1,2, \cdots$. We shall show that this alternative is impossible by proving that it implies $\alpha \omega \leqq \sigma$.

For each $n$ there exists a similarity transformation of $B_{n} \cup A_{n+1}$ into $D$. This implies that for each $n$, there exists a similarity transformation $f_{n}$ of $B_{n}$ into $D_{m(n)}$, where $m(n)$ is some positive integer. Let $k_{n}$ be the smallest integer for which there exists a similarity transformation, say $g_{n}$, of $B_{n}$ into $D$, so that $g_{n}\left(B_{n}\right) \subseteq D_{k_{n}}$. Since $\alpha n<\alpha(n+1)$, it follows that $n \leqq k_{n}$. Next, suppose that $g_{n}\left(A_{j+1}\right) \cap A_{i} \neq \phi$ for some $i$ and $j$, where $0<i<j<n$. Then $g_{n}\left(B_{j}\right) \subseteq B_{i}$. This implies $\alpha j \leqq \alpha i$, which is a contradiction. Therefore

$$
g_{n}\left(A_{j+1}\right) \cap A_{i}=\phi \text { for } 0<i<j<n .
$$

Denote by $h(x)$ the following function:

and

$$
h(x)=g_{k(1)}(x) \text { for } x \text { in } A_{k(1)} \text {, where } k(1)=1 \text {; }
$$

$h(x)=g_{k(n+1)}(x)$ for $x$ in $A_{k(n+1)}$, where $k(n+1)=k_{k(n)}+2$ for $n \leqq 1$. If $G=\bigcup_{1 \leqq n<\omega} A_{k(n)}$, then $\bar{G}=\alpha \omega$. Furthermore, $h$ is a similarity transformation of $G$ into $D$. Thus $\alpha \omega \leqq \sigma$.

Finally, suppose that $\sigma$ is incomparable with $\alpha n_{0}$ for some positive integer $n_{0}$. Let $F=D \cup B_{n_{0}}$ and $\tau=\bar{F}$. Since there can be no similarity transformation of $F$ into $B_{n_{0}}$ as $\bar{B}_{n_{0}}$ and $\bar{D}$ are incomparable, it follows that $\alpha n_{0}<\tau$. To show that $\tau<\alpha \omega$ assume the contrary. Let $f$ be a similarity transformation of $B$ into $F$. Let $G=F-B_{n_{0}}$, and $B^{*}=\{x \mid f(x) \in G\}$. Note that $G \subseteq D$. Since $\alpha n_{0}<\alpha \omega, B^{*} \neq \phi$. If $x_{0}$ is any element of $B^{*}$, then

$$
\left\{x \mid x \leqq x_{0}, x \in B\right\} \subseteq B^{*} .
$$

Consequently $\bar{B}^{*} \equiv \alpha \omega$. It follows that

$$
\alpha \omega \equiv \bar{B}^{*} \leqq \bar{G} \leqq \bar{D}=\sigma<\alpha \omega .
$$

From this contradiction we conclude that $\tau<\alpha \omega$. Q.E.D.

The condition that $\alpha<\alpha 2$ occurs quite frequently. For example, consider $\alpha=\bar{A}$, where $A$ has a fixed point $z_{0}\left({ }^{3}\right)$. Let $A_{1}$ and $A_{2}$ be the same as in

(3) See $\$ 2$. 
Lemma 1.3 , and $x_{0}$ a point in $A_{1}$ which is the image point of $z_{0}$ under some similarity transformation of $A$ onto $A_{1} . x_{0}$ is a fixed point of $A_{1}\left({ }^{4}\right)$. Let $f$ be a similarity transformation of $A_{1}$ onto $A_{2}$ and suppose that $\bar{A}_{1} \equiv \overline{A_{1} \cup A_{2}}$. If $g$ is a similarity transformation of $A_{1} \cup A_{2}$ into $A_{1}$, then $g\left(x_{0}\right)=x_{0}$. The element $f\left(x_{0}\right)$ is in $A_{2}$, so that $g f\left(x_{0}\right) \neq g\left(x_{0}\right)=x_{0}$. Since $g f$ is a similarity transformation of $A_{1}$ into itself, the fact that $g f\left(x_{0}\right) \neq x_{0}$ contradicts $x_{0}$ being a fixed point of $A_{1}$. Therefore $\bar{A}_{1} \equiv \overline{A_{1} \cup A_{2}}$ is false, so that $\alpha<\alpha 2$. Summarizing we get

ThEOREM 1.4. If $A$ has a fixed point, and $\sigma<\bar{A} \omega$, then there exists an order type $\tau$ for which $\sigma<\tau<\bar{A} \omega$.

Suppose that $\bar{E} \equiv \alpha \omega$, where $\alpha \equiv \alpha 2$. Does problem (P) have an affirmative solution? In case $\alpha \equiv \lambda$, problem (P) has been shown to have a positive answer $[6$, p. 260]. Consider when $\alpha \equiv \eta$. Here too we shall see that problem (P) possesses an affirmative answer [Theorem 1.6].

LemmA 1.4. Let $\bar{E} \equiv \alpha$, where $\alpha \equiv \alpha 2$. Then $\alpha \equiv \alpha \eta$.

Proof. Since $\alpha \equiv \alpha 2 \equiv(\alpha 2) 2 \equiv \alpha 4$, it follows that $\alpha \equiv \alpha 3$. Let $C$ and $D$ be two disjoint subsets of $E$ such that $\bar{C}=\bar{D}=\alpha$ and $x<y$ for any elements $x$ and $y$ of $C$ and $D$ respectively. Let $C_{0}, A_{0}$, and $D_{0}$ be three disjoint subsets of $C$ such that $\bar{C}_{0}=\bar{A}_{0}=\bar{D}_{0}=\alpha$ and $x<y<z$ for any elements $x, y$, and $z$ of $C_{0}$, $A_{0}$, and $D_{0}$ respectively. This is possible as $\alpha \equiv \alpha 3$. Repeat the same procedure in $D$, obtaining the sets $C_{1}, A_{1}$, and $D_{1}$. We continue by induction. Suppose that the sets

$$
C_{a_{0}} \cdots a_{k}, A_{a_{0}} \cdots a_{k}, \text { and } D_{a_{0}} \cdots a
$$

have been obtained for $k \leqq n$ and $a_{i}=0,1$. Let

$$
X=C_{a_{0} \cdots a_{n} 0}, Y=A_{a_{0} \cdots a_{n} 0} \text {, and } Z=D_{a_{0} \cdots a_{n} 0}
$$

be three disjoint subsets of $C_{a_{0}} \cdots a_{n}$, such that $\bar{X}=\bar{Y}=\bar{Z}=\alpha$ and $x<y<z$ for any elements $x, y$, and $z$ of $X, Y$, and $Z$ respectively. Repeat the procedure in $D_{a_{0}} \cdots a_{n}$, obtaining the sets

$$
C_{a_{0}} \ldots a_{n} 1, A_{a_{0} \cdots a_{n} 1} \text {, and } D_{a_{0} \cdots a_{n} 1} \text {. }
$$

Let $X_{a_{0} \cdots a_{n}}$ be some definite element of $A_{a_{0} \cdots a_{n}}$, and denote by $G$ the set

$$
G=\left\{x_{a_{0}} \ldots a_{n} \mid a_{i}=0,1 ; n<\omega\right\} .
$$

The set $G$ has the following three properties:

( $\alpha$ ) the power of $G$ is $\aleph_{0}$;

( $\beta$ ) $G$ is dense, i.e., if $x$ and $y$ are any two elements of $G$, then there exists a third element $z$ of $G$ which lies between $x$ and $y$; and

$(\gamma) G$ has no first and no last element.

(4) See (I) of $\$ 2$. 
The set $G$ therefore has order type $\eta$, since the above three properties are a well known characterization of any set which is similar to the rational numbers. Consider the subset of $E$,

$$
H=\bigcup_{n<\omega} A_{a_{0} \cdots a_{n}} .
$$

Since $G$ has order type $\eta$ and $H$ is obtained from $G$ by replacing each element of $G$ by a set of order type $\alpha, \bar{H}=\alpha \eta$. Thus, as $H \subseteq E, \alpha \eta \leqq \alpha$. Consequently $\alpha \eta \equiv \alpha$.

\section{Using Lemma 1.4 we now prove}

TheOREM 1.5. Let $\bar{E} \equiv \alpha \omega$, where $\alpha \equiv \alpha 2$. Let $\sigma<\alpha$, where $\sigma<\sigma 2$. Then there exists an order type $\tau$ such that $\sigma<\tau<\bar{E}$.

Proof. $\alpha \omega \equiv(\alpha \eta) \omega \equiv \alpha(\eta \omega) \equiv \alpha \eta \equiv \alpha$. Consider the order type $\tau=\sigma 2$. Either $\sigma 2<\alpha$ or $\sigma 2 \equiv \alpha$. It shall be shown that the latter alternative is impossible, thus implying that $\sigma<\tau<\alpha$. Consider the two disjoint sets $A$ and $B$, where $\bar{A}=\bar{B}=\sigma$ and $x<y$ for any two elements $x$ and $y$ of $A$ and $B$ respectively. Let $C$ and $D$ be two disjoint subsets of $A \cup B$ such that $\bar{C}=\bar{D}=\alpha$, and $u<v$ for any two elements $u$ and $v$ of $C$ and $D$ respectively. Clearly, either $C \subseteq A$, or $D \subseteq B$. Hence $\alpha \leqq \sigma$. But this contradicts our assumption that $\sigma<\alpha$. Q.E.D.

Suppose that $\alpha \equiv \alpha 2$ and $\sigma<\alpha$, where $\sigma \equiv \sigma 2$. If there exists an order type $\mu<\alpha$ which is incomparable with $\sigma$, then as is easily seen, $\sigma<\sigma+\mu<\alpha$. Now suppose that in addition to $\alpha \equiv \alpha 2$ and $\sigma<\alpha$, where $\sigma \equiv \sigma 2, \alpha$ has the property that $\alpha \equiv \delta \eta$, where $\delta<\alpha$. Since $\delta<\sigma$ implies that $\alpha \equiv \delta \eta \leqq \sigma \eta \equiv \sigma$, the only two alternatives which can arise are either (a) $\sigma<\delta$, or (b) $\sigma$ is incomparable with $\delta$. If $\sigma<\delta$, then $\sigma<\delta<\alpha$. If the latter possibility occurs, then as remarked above, $\sigma<\sigma+\delta<\alpha$. Summarizing the preceding results we see that if $\bar{E} \equiv \alpha \omega$ and $\sigma<\bar{E}$, then there always exists an order type $\tau$ for which $\sigma<\tau<\bar{E}$, except possibly when the following four conditions hold simultaneously:

(1) $\sigma \equiv \sigma 2$;

(2) $\alpha \equiv \alpha 2$;

(3) $\alpha \neq \equiv\rangle$ for $\delta<\alpha\left({ }^{5}\right)$;

(4) $\sigma$ is comparable with each order type $\mu$ which is $<\alpha$.

Let $\sigma$ be any order type $\langle\eta$. Since $\sigma \equiv \sigma \eta$ must be false it follows that $\sigma<\sigma 2$. From Theorem 1.5 and the fact that $\eta \eta \equiv \eta$ we deduce

TheOREm 1.6. If $\sigma<\eta$, then there exists an order type $\tau$ for which $\sigma<\tau<\eta$.

We now present an alternative proof of Theorem 1.6 which depends on a characterization of those sets $E$ for which $\bar{E} \equiv \eta$ (Theorem 1.7).

Definition. A set $E$ is a universal set for each denumerable ordinal type if, for every denumerable ordinal number $\alpha, E$ contains a subset of ordinal type $\alpha$.

(5) By $\alpha \neq \equiv \eta$ is meant that $\alpha \equiv \delta \eta$ is false. 
Lemma 1.5. If $E$ is a denumerable set which is a universal set for all denumerable ordinal types, then there exists a point $y$ of $E$ so that each of the two sets

$$
A(y)=\{x \mid x<y, x \in E\} \quad \text { and } B(y)=\{x \mid x>y, y \in E\}
$$

is a universal set for each denumerable ordinal type.

Proof. By universal set we shall mean universal set for each denumerable ordinal type. Assume the conclusion of the lemma is false, i.e., for each element $y$ of $E$, at least one of the sets, $A(y)$ or $B(y)$, is not a universal set. We shall show that this leads to a contradiction. Well order the elements of $E$ into the sequence $\left\{y_{n}\right\}, n<\omega$. This is certainly possible since the power of $E$ is $\boldsymbol{\aleph}_{0}$. Denote by $x_{0}$ the element $y_{0}$. Suppose that $A\left(x_{0}\right)$ contains no subset of ordinal type $\xi$, and $B\left(x_{0}\right)$ contains no subset of ordinal type $\nu$. Then

$$
E=A\left(x_{0}\right) \cup\left\{x_{0}\right\} \cup B\left(x_{0}\right),
$$

and thus contains no subset of ordinal type $\xi+1+\nu$. But this contradicts the fact that $E$ is a universal set. Consequently one of the two sets, $A\left(x_{0}\right)$ or $B\left(x_{0}\right)$, call it $C_{0}$, is a universal set, and the other set, call it $D_{0}$, is not. Let $\gamma_{0}$ be the smallest ordinal for which $D_{0}$ contains no subset of ordinal type $\gamma_{0}$. We continue by induction. Suppose that for each $k \leqq n$, the point $x_{k}$, the sets $C_{k}$ and $D_{k}$, and the ordinal number $\gamma_{k}$ have been defined such that $C_{k}$ is a universal set and $D_{k}$ is not. Let $x_{n+1}$ be the first point of $E$ which is in $C_{n}$. Let $A_{n+1}=\left\{x \mid x<x_{n+1}, x \in C_{n}\right\}$ and $B_{n+1}=\left\{x \mid x>x_{n+1}, x \in C_{n}\right\}$. Then one of the two sets, either $A_{n+1}$ or $B_{n+1}$, call it $C_{n+1}$, is a universal set and the other, call it $D_{n+1}$, is not. This is so by the following argument. Since $C_{n}$ is a universal set, one of the two sets, $A_{n+1}$ or $B_{n+1}$, must also be a universal set. If both were universal sets, then obviously both $A\left(x_{n+1}\right)$ and $B\left(x_{n+1}\right)$ would be universal sets. But we have assumed that this is not so. Define $\gamma_{n+1}$ to be the smallest ordinal for which $D_{n+1}$ contains no subset of order type $\gamma_{n+1}$.

Let $G_{n}=D_{n} \cup\left\{x_{n}\right\}$. Note that $G_{n+1} \subseteq C_{n}$. Consider the set $F=\cup_{n<\omega} G_{n}$. Suppose that $y$ is an element of $E$, say $y_{m}$. From the definition of the sets $D_{n}$ and the manner in which the points $x_{n}$ were defined, it follows that at the $(m+2)$ th stage, either $y_{m}$ is in a set $D_{j}$ or is an element $x_{i}$, where $j \leqq m, i \leqq m$. Therefore $F=E$. Let $\gamma$ be the smallest ordinal number which is greater than each ordinal number $\gamma_{k}+1$. Since the power of each $\gamma_{k}$ is $\leqq \boldsymbol{N}_{0}$, and there are only an enumerable number of $\gamma_{k}$, the power of $\gamma$ is $\boldsymbol{\aleph}_{0}$.

Let $H=\left\{x_{n} \mid n<\omega\right\}$. Consider the order type $\mu$ of $H$. Since $G_{n+1} \subseteq C_{n}, \mu$ has one of the forms:

(1) $n+\omega^{*} \quad(0<n<\omega)$

$\omega+n$

$(0<n<\omega)$; or

(3) $\omega+\omega^{*}$.

Now consider any well ordered subset $Z$ of $E$. Denoting by $Z_{n}$ the set $G_{n} \cap Z$ we see that $Z=\bigcup_{0 \leqq n<\omega} Z_{n}$. Since $Z_{n}$ is a subset of $G_{n}$, the order type of $Z_{n}$ cannot exceed $\gamma_{n}$. Let $v_{n}$ be any element of $Z_{n}$ and $v_{m}$ any element of $Z_{m}$, 
where $n \neq m$. Then $v_{n}<v_{m}$ or $v_{n}>v_{m}$, according as $x_{n}<x_{m}$ or $x_{n}>x_{m}$. Combining these two facts we see that the order type of $Z=U Z_{n}$ cannot exceed $\gamma \omega 2$. Since $Z$ was any well ordered subset of $E$, this contradicts the fact that $E$ is a universal set. From this contradiction we are forced to conclude that there exists a point $y$ of $E$ for which each of the two sets, $A(y)$ and $B(y)$, is a universal set. Q.E.D.

REMARK. The cardinality condition on the set $E$ in Lemma 1.5 is needed in order that the conclusion be true. Witness the set of all ordinals $<\omega_{1}$.

Using Lemma 1.5 we now arrive at Shepherdson's result [4, p. 304].

Lемма 1.6. If $E$ is a denumerable set which is a universal set for each denumerable ordinal type, then $E$ contains a subset $D$ which is similar to the set of rational numbers.

Proof. An element $y$ of $G$ shall be called an " $r$-point" (of $G$ ) if each of the two sets, $\{x \mid<y, x \in G\}$ and $\{x \mid x>y, x \in G\}$, is a universal set for each denumerable ordinal type. In terms of $r$-points Lemma 1.5 can be stated as "Every denumerable set which is a universal set for each denumerable ordinal type possesses an $r$ point."

Let $y$ be an $r$-point of $E$ and

$$
A_{0}=\{x \mid x<y, x \in E\} \quad \text { and } A_{1}=\{x \mid x>y, x \in E\} .
$$

Let $x_{0}$ be an $r$-point of $A_{0}$ and $x_{1}$ an $r$-point of $A_{1}$. We continue by induction. Suppose that the elements $x_{a_{0} a_{1} \cdots a_{k}}$ and the sets $A_{a_{0} a_{1} \cdots a_{k}}$, where $a_{i}=0,1$, and $k \leqq n$, have been defined. Let

$$
A_{a_{0} a_{1} \ldots a_{n} 0}=\left\{x \mid x<x_{a_{0} a_{1} \cdots a_{n}}, x \in A_{a_{0} a_{1} \cdots a_{n}}\right\}
$$

and

$$
A_{a_{0} a_{1}} \cdots a_{n} 1=\left\{x \mid x>x_{a_{0} a_{1} \cdots a_{n}}, x \in A_{a_{\mathrm{c}} a_{1} \cdots a_{. .}}\right\} \text {. }
$$

Let $x_{a_{0} a_{1} \cdots a_{n} a_{n+1}}$ be an $r$-point of $A_{a_{0} a_{1} \cdots a_{n+1}}$. Denote by $D$ the set $D$ $=\left\{x_{a_{0} a_{1} \cdots a_{n}} \mid 0 \leqq n<\omega\right\}$. The set $D$ has the following three properties:

$(\alpha)$ the power of $D$ is $\boldsymbol{N}_{0}$;

$(\beta) D$ is dense;

$(\gamma) D$ has no first and no last element.

The set $D$ therefore has order type $\eta$, and so satisfies the conclusion of the lemma.

As a corollary we have

THEOREM 1.7. A necessary and sufficient condition that $\bar{E} \equiv \eta$ is that $E$ be a denumerable set which is a universal set for each denumerable ordinal type.

We now present another proof of Theorem 1.6( $\left.{ }^{6}\right)$.

Proof. Let $E$ denote the set of rational numbers and $y$ an element of $E$. Let

(8) I am indebted to the referee for this proof. 
$M=\{x \mid x<y, x \in E\}$ and $N=\{x \mid x>y, x \in E\}$. Let $A$ be a subset of $M$ such that $\bar{A}=\sigma$. By Theorem 1.7, for some ordinal number $\alpha$, no subset of $A$ is of ordinal type $\alpha$. Let $B$ be a subset of $N$ such that $\bar{B}=\alpha$. Let $F=A \cup B$ and $\tau=\bar{F}$. Since $B$ is not similar to any subset of $A, \bar{A}<\bar{F}$. The set $F$ in turn contains no subset of order type $\eta$. Hence $\bar{F}<\eta$. On combining these results we get $\bar{A}<\bar{F}<\eta$.

2. Fixed points.

Definition. An element $x_{0}$ of $E$ is called a "fixed point" of $E$ if $f\left(x_{0}\right)=x_{0}$ for every similarity transformation of $E$ into itself.

Definition. A subset $A$ of $E$ is called a "set of consecutive elements" of $E$ if, for each pair of elements of $A, a$ and $b$, where $a<b$, the set

$$
\{x \mid a \leqq x \leqq b, x \in E\}
$$

is finite, and is also a subset of $A$. If $A$ consists of only two elements, $a$ and $b$, then $a$ and $b$ are called "consecutive."

The following statements are easily verified:

(I) Let $\bar{E} \equiv \bar{F}$. If $h$ is a similarity transformation of $E$ into $F$, and $x_{0}$ is a fixed point of $E$, then $h\left(x_{0}\right)$ is a fixed point of $F$.

(II) Let $\bar{E} \equiv \bar{F}$. If $x_{0}$ is a fixed point of $E$, and $f$ and $g$ are any two similarity transformations of $E$ into $F$, then $f\left(x_{0}\right)=g\left(x_{0}\right)$.

(III) If $\bar{E} \equiv \sigma \mu$, where $\sigma$ is any order type and $\mu$ is any limit number, then $E$ has no fixed point. $\mu=\omega$ provides an important instance.

(IV) If $x$ and $y$ are consecutive elements of $E$ and $x$ is fixed, then $y$ is also fixed.

(V) If $x_{0}$ is a fixed element of $E$, then it is impossible to have an $\omega, \omega^{*}$, or $\omega^{*}+\omega$ sequence of consecutive elements of $E$ which contains $x_{0}$.

(VI) Let $x_{0}$ and $x_{1}$ be two consecutive elements of $E$. If $x_{1}$ is fixed in $E$ and $A=E-\left\{x_{1}\right\}$, then $x_{0}$ is a fixed point of $A$.

We may characterize fixed points by

THEOREM 2.1. A necessary and sufficient condition that $x_{0}$ be a fixed point of $E$ is that $\overline{E-\left\{x_{0}\right\}}<\bar{E}$.

Proof. If $x_{0}$ is fixed, then each similarity transformation of $E$ into itself takes $x_{0}$ into $x_{0}$. Thus there is no similarity transformation of $E$ into $E$ $-\left\{x_{0}\right\}$, i.e., $\overline{E-\left\{x_{0}\right\}}<\bar{E}$. Now suppose that $\overline{E-\left\{x_{0}\right\}}<\bar{E}$. For any similarity transformation $f$ of $E$ into itself such that $f\left(x_{0}\right) \neq x_{0}$, say $f\left(x_{0}\right)>x_{0}$, let $g$ be defined by $g(x)=x$ for $x<x_{0}$ and $g(x)=f(x)$ for $x \geqq x_{0}$. Then $g$ takes $E$ into $E-\left\{x_{0}\right\}$, which is a contradiction. Consequently $x_{0}$ is a fixed point of $E$.

The partial ordering of order types is akin to the partial ordering of dimension types as defined by Fréchet. For the former, the functions considered are similarity transformations, whereas in the latter, the functions considered are homeomorphisms. Theorems stated for dimension type frequently have a counterpart for order type, and conversely. In [5, pp. 122-123] Sierpiński 
has given an example of a set $E$ and a point $x_{0}$ in $E$ for which $E-\left\{x_{0}\right\}$ $<E$ (" $<$ " for dimension types). Furthermore, there exist dimension types $\sigma$ and $\tau$ such that $E-\left\{x_{0}\right\}<\sigma<\tau<E$ (" $<$ " for dimension types). A consequence of the following theorem is that such a result cannot occur for " $<$ " for order types.

THEOREM 2.2. If $\left\{x_{0}, x_{1}, \cdots, x_{n}\right\}$ is any set of $n+1$ consecutive points of $E$, if $x_{0}$ is any fixed point of $E$, and if $A=E-\left\{x_{i} \mid i \leqq n\right\}$, then there are precisely $n$ distinct order types between $\bar{A}$ and $\bar{E}$.

Proof. We shall verify the theorem for $n=0$, and simultaneously, by assuming it true for $k \leqq n-1$, prove it for $k=n$, where $n>0$.

For $i=0, \cdots, n$, denote by $A_{i}$ the set $A \cup\left\{x_{j} \mid j \leqq i\right\}$. From (VI) and 'Theorem 2.1 , it follows that $\bar{A}<\bar{A}_{0}<\bar{A}_{1}<\cdots<\bar{A}_{n}=\bar{E}$, so that there are at least $n$ distinct order types between $\bar{A}$ and $\bar{E}$. To complete the theorem we shall show that if $\bar{A}<\tau<\bar{E}$, then $\tau \equiv A_{i}$ for some $i$. Consider any order type $\tau$ for which $\bar{A}<\tau<\bar{E}$ and $\tau$ is distinct from each $A_{i}, i=0,1,2, \cdots, n-1$. Let $B$ be a subset of $E$ for which $\bar{B}=\tau$, and $g$ a similarity transformation of $A$ into $B$. The set $B$ contains each element $x_{i}, i \leqq n$. This follows from the following argument. For $n=0$, if $B$ does not contain $x_{0}$, then $B \subseteq A$. This contradicts the fact that $\bar{A}<\bar{B}$. For $n>0$ suppose that $B$ does not contain the element $x_{s}$. Let $f$ be a similarity transformation of $B \cap\left\{x_{i} \mid i \leqq n\right\}$ into $\left\{x_{i} \mid\right.$ $i \leqq n-1\}$, and $h$ the function defined by $h(x)=x$ for $x$ in $B-\left\{x_{i} \mid i \leqq n\right\}$ and $h(x)=f(x)$ for $x$ in $B \cap\left\{x_{i} \mid i \leqq n\right\}$. Then $h(B) \subseteq A_{n-1}$, so that

$$
\bar{A}<\bar{B}=\overline{(h) B} \leqq \bar{A}_{n-1} \text {. }
$$

By our induction hypothesis, $\bar{B} \equiv \bar{A}_{i}$ for some $i \leqq n-1$.

Denote by $G$ the set $\left\{x \mid x<x_{0}, x \in E\right\}$ and by $H$ the set $\left\{x \mid x>x_{n}, x \in E\right\}$. Now suppose that

$$
g(G) \subseteq G \text { and } g(H) \subseteq H .
$$

If $h$ is the function defined by $h(x)=g(x)$ for $x$ in $G \cup H$ and $h\left(x_{i}\right)=x_{i}$, then since the elements $x_{i}$ are in $B$, it follows that $h$ is a similarity transformation of $E$ into $B$. This again is a contradiction. Therefore we may suppose that the two relations in (*) do not hold. Then one of them, either $g(G) \subseteq G$ or $g(H)$ $\subseteq H$, is false. We shall discuss the case for which the latter relation is false, a similar treatment being possible for the former. Two alternatives now arise. Either $g(y)<x_{0}$ for some $y$ in $H$, or for no $y$ in $H$ is $g(y)<x_{0}$. Consider the former situation first. Let $K=\left\{x \mid x \in H, g(x)<x_{0}\right\}$. Denote by $h$ the function defined by $h(x)=g(x)$ for $x$ in $H, h\left(x_{n-i}\right)=g^{2+i}\left(y_{0}\right), i=0,1,2, \cdots, n$, and $h(x)=g^{2+n}(x)$ for $x$ in $G$, where $y_{0}$ is some element in $K$, and $g^{k}\left(y_{0}\right)$ is the element $g\left[g^{k-1}\left(y_{0}\right)\right]$. The function $h(x)$ is a similarity transformation of $E$ into $E$ for which $h\left(x_{0}\right) \neq x_{0}$. This contradicts $x_{0}$ being a fixed point. Now consider the latter case, i.e., for no $y$ in $H$ is $g(y)<x_{0}$. Since $x_{0}$ is fixed, by 
(V) the sequence of consecutive points in $E$

$$
\cdots<y_{1}^{*}<y_{0}^{*}<x_{0}<x_{1}<\cdots x_{n}<y_{0}<y_{1}<\cdots
$$

is finite. Let $y_{p}$ be the last term and $y_{q}^{*}$ the first, i.e., there is no point $y_{p+1}$ $\left(y_{q+1}^{*}\right)$ of $E$ which has the two properties

$$
\begin{aligned}
& y_{p}<y_{p+1}\left(y_{q+1}^{*}<y_{q}^{*}\right) \text {, and } \\
& \text { for no element } z \text { in } E \text { is } y_{p}<z<y_{p+1}\left(y_{q+1}^{*}<z<y_{q}^{*}\right) .
\end{aligned}
$$

Let $L=H-\left\{y_{i} \mid i \leqq p\right\}$. If $z_{0}$ is any element of $L$, then points $z_{j}$ in $L$ exist for which

$$
\cdots<z_{1}<z_{0} \text {. }
$$

As $g(H)$ is not contained in $H$, for some $\bar{y}$ in $H, g(\bar{y})=x_{i_{0}}$. Thus, if $z$ is in $L$, then $g(z)$ is in $L$. It also follows that for no $y$ in $G$ is $g(y)>x_{i_{0}}$. By a similar argument, if $M=G-\left\{y_{i}^{*} \mid i \leqq q\right\}$, then for $y$ in $M, g(y)$ is also in $M$. Let

$$
N=\left\{x_{i} \mid i \leqq n\right\} \cup\left\{y_{j} \mid j \leqq p\right\} \cup\left\{y_{j}^{*} \mid j \leqq q\right\} .
$$

If $N \subseteq B$, then let $h$ be the function defined by $h(x)=g(x)$ for $x$ in $L \cup M$ and $h(x)=x$ for $x$ in $N-\left\{x_{n}\right\}$. For $n=0, h(G) \subseteq G$ and $h(H) \subseteq H$, a fact which has previously been proved impossible. For $n>0$, the similarity transformation $h$ takes $A_{n-1}$ into $B$, so that $\bar{A}_{n-1} \leqq \bar{B}<\bar{A}_{n}$. By our induction hypothesis, $\bar{B}$ $\equiv \bar{A}_{n-1}$. If $N$ is not contained in $B$, then let $f$ be a similarity transformation of $N \cap B$ into $N-\left\{x_{n}\right\}$. Denote by $h$ the function defined by $h(x)=x$ for $x$ in $B \cap(L \cup M)$ and $h(x)=f(x)$ for $x$ in $B \cap N$. For $n=0, \bar{B}=\overline{h(B)} \leqq \bar{A}$, which is a contradiction. For $n>0, \bar{A}<\bar{B}=\overline{h(B)} \leqq \bar{A}_{n-1}$. Thus $\bar{B} \equiv \bar{A}_{i}$ for some $i \leqq n-1$.

We now have treated all possibilities, and have seen that in every case we are led to a contradiction. Thus whenever $\bar{A}<\tau<\bar{A}_{n}$, then $\tau \equiv \bar{A}_{i}$ for some $i$. By mathematical induction the theorem becomes true for all non-negative integers.

REMARK. The proof of the above theorem would be trivial if the following result were true: "Let $\bar{A}<\bar{C}, A \subset C$, and $\bar{A}<\tau<\bar{C}$. Then a set $B$ can be found, $A \subset B \subset C$, for which $\bar{B} \equiv \tau$." Unfortunately such a set $B$ cannot always be found. This is so in the following example. Let $A=\{\xi \mid \omega \leqq \xi<\omega 2\}$ and $C$ $=\{\xi \mid \xi<\omega 2\}$. Then $\bar{A}<\omega+1<\bar{C}$. However, there is no subset of $C$ which contains $A$ and whose order type is $\omega+1$.

From Theorems 2.1 and 2.2 we infer

Corollary 2.1. If $\overline{E-\left\{x_{0}\right\}}<\bar{E}$, then there is no order type $\tau$ for which $\overline{E-\left\{x_{0}\right\}}<\tau<\bar{E}$.

By a proof similar to that of Theorem 2.2 we can show 
Corollary 2.2. Let $x_{0}$ be a fixed two-sided limit point of $E$ in the order topology [1, p. 39]. If $g$ is a similarity transformation of $E-\left\{x_{0}\right\}$ into $E$, then $g$ can be extended to become a similarity transformation of $E$ into $E$, i.e., if

$G=\left\{x \mid x<x_{0}, x \in E\right\}$ and $H=\left\{x \mid x>x_{0}, x \in E\right\}$, then $g(G) \subseteq G$ and $g(H) \subseteq H$.

The conclusion of Corollary 2.2 is no longer valid if the phrase " $x$ is a twosided limit point" is removed. One has only to consider a simply ordered set of five elements.

Corollary 2.3. If $x_{0}$ is a fixed point of $E, A=\left\{x \mid x \leqq x_{0}, x \in E\right\}, B$ $=\left\{x \mid x>x_{0}, x \in E\right\}$, and $n$ is a positive integer, then there are precisely $n$ distinct order types $\tau$ for which $\bar{E}<\tau<\bar{A}+n+1+\bar{B}$.

By a demonstration quite similar to that given in the proof of Theorem 2.2 , we can also show

Corollary 2.4. If $x_{0}$ is a fixed point of $E, A=\left\{x \mid x \leqq x_{0}, x \in E\right\}$, and $B$ $=\left\{x \mid x>x_{0}, x \in E\right\}$, then there are precisely $\aleph_{0}$ distinct order types $\tau$ for which $E<\tau<\bar{A}+\omega+\bar{B}$.

We shall call the element $x_{0}$ a "right-sided" ("left-sided") $c$-condensation point of the linear set $E$ if for each element $x_{1}$ in $R$ such that $x_{1}<x_{0}\left(x_{1}>x_{0}\right)$, the power of the set

$$
\left\{x \mid x_{1}<x<x_{0}, x \in E\right\} \quad\left(\left\{x \mid x_{0}<x<x_{1}, x \in E\right\}\right)
$$

is $2^{\aleph_{0}}$. A point $x_{0}$ which is both a left- and a right-sided $c$-condensation point of $E$ will be called a $c$-condensation point of $E$, and a point $x_{0}$ which is either a left- or a right-sided $c$-condensation point a one-sided $c$-condensation point of $E$.

We shall say that a linear set $E$ has "property A" if the power of $E$ is $2^{N_{0}}$, and no two disjoint subsets of $E$ of power $2^{\aleph_{0}}$ each are similar.

TheOREM 2.3. Let $E$ have property $\mathrm{A}$ and $D$ be any subset of $E$ of power $2^{\mathrm{N}_{0}}$. If $x_{0}$ is any c-condensation point of $D$ which belongs to $D$, then $x_{0}$ is a fixed point of $D$.

Proof. Assume the contrary, i.e., for some similarity transformation $f$ of $D$ into itself, $f\left(x_{0}\right) \neq x_{0}$. Suppose that $f\left(x_{0}\right)>x_{0}$. Let $B=\left\{x \mid x \geqq x_{0}, x \in D\right\}$ and $g$ be the similarity transformation of $B$ into $B$ defined by $g(x)=f(x)$ for $x$ in $B$. Since $x_{0}$ is a $c$-condensation point of $D$, the power of the set $C=B-g(B)$ is $2^{N_{0}}$. The set $C$ is thus similar to a subset of $g(B)$, i.e., $C$ and $g(C)$ are two disjoint subsets of $E$, of power $2^{N_{0}}$ each, which are similar. This contradicts the fact that $E$ has property A. Similarly, by assuming that $f\left(x_{0}\right)<x_{0}$, we are led to a contradiction. It follows that we must have $f\left(x_{0}\right)=x_{0}$, so that $x_{0}$ is indeed a fixed point of D. Q.E.D. 
If the words " $c$-condensation point" are replaced by "one-sided $c$-condensation point," then the conclusion of Theorem 2.3 is no longer valid.

From Theorem 2.1, Corollary 2.1, and Theorem 2.3 we infer

Corollary 2.5. If $E$ has property $\mathrm{A}$, if $B$ is a subset of $E$ of power $2^{\mathrm{No}_{0}}$, and if $x_{0}$ is a c-condensation point of $B$ which belongs to $B$, then $\overline{B-\left\{x_{0}\right\}}<\bar{B}$, and there is no order type $\tau$ for which $\overline{B-\left\{x_{0}\right\}}<\tau<\bar{B}$.

COROLlaRy 2.6. Let $E$ have property $\mathrm{A}$. If $B$ is the set of $c$-condensation points of $E$ which belong to $E$, then $B$ is exact.

In Theorem 5 of [3] it has been shown that if $E$ is any linear set of power $2^{\aleph_{0}}$, then $E$ contains a subset $A$ which has property A. From this we deduce

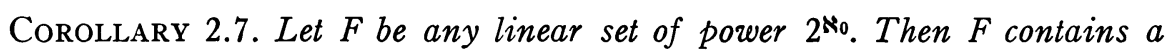
subset $E$ of power $2^{N_{0}}$ such that if $A$ is any subset of $E$ of power $2^{N_{0}}$, then $A$ contains $2^{\mathrm{s}_{0}}$ distinct points $x_{0}$ which have the following two properties: (1) $\overline{A-\left\{x_{0}\right\}}<\bar{A}$, and (2) there is no order type $\tau$ for which $\overline{A-\left\{x_{0}\right\}}<\tau<\bar{A}$.

According to Theorem 4 in [3], if $E$ is any linear set of power $2^{\mathrm{N}_{0}}$, then there exists an exact order type $\rho$, of power $2^{\aleph_{0}}$, such that $\rho<\bar{E}$. In Theorem 2.4 we shall show that there exists an exact order type $\tau$ for which $\bar{E}<\tau<\lambda$. This strengthens the result due to Sierpinski which states that if $\bar{E}<\lambda$, then there exists an order type $\tau$ such that $\bar{E}<\tau<\lambda[6$, p. 260].

THEOREM 2.4. If $E$ is any set of power $2^{\aleph_{0}}$ such that $\bar{E}<\lambda$, then there exists an exact set $B$ which is dense in $R$ and for which $\bar{E}<\bar{B}<\lambda$. Furthermore, each point of $B$ is a c-condensation point of $B$.

Proof. Without loss of generality we shall suppose that $E$ is a linear set. Denote by $F=\{f\}$ the set of those similarity transformations of $R$ into $R$ which are not the identity transformation. Well order the elements of $R$ and $F$ into the two sequences $\left\{x_{\xi}\right\}, \xi<\theta$, and $\left\{f_{\xi}\right\}, \xi<\theta$, where each element of $F$ appears $2^{\aleph_{0}}$ times in the latter sequence. By transfinite induction we shall define two disjoint sequences of $R,\left\{p_{\xi}\right\}, \xi<\theta$, and $\left\{q_{\xi}\right\}, \xi<\theta$.

Since $f_{\xi}$ is not the identity transformation, the set

$$
G_{\xi}=\left\{x \mid f_{\xi}(x) \neq x\right\}
$$

contains an interval $J_{\xi}$. Let $f^{*}$ denote the inverse function of $f$. Let $p_{0}$ be the first element in $J_{0}-f_{0}^{*}(E)$. Such an element certainly exists since $\bar{E}<\lambda$ implies that $E$ does not contain $f_{0}\left(J_{0}\right)$. Let $q_{0}=f_{0}\left(p_{0}\right)$. Now suppose that $\left\{p_{\xi}\right\}, \xi<\mu$, and $\left\{q_{\xi}\right\}, \xi<\mu$, have been defined for $\mu<\theta$. By Lemma 3 of [6], if

$$
A_{\mu}=E \cup B_{\mu} \text {, where } B_{\mu}=\left\{p_{\xi} \mid \xi<\mu\right\} \cup\left\{q_{\xi} \mid \xi<\mu\right\},
$$

then $\bar{A}_{\mu}<\lambda$. Thus $A_{\mu}$ does not contain $f_{\mu}\left(J_{\mu}\right)$. In fact, we see that the power of $C_{\mu}=J_{\mu}^{*}-f_{\mu}^{*}\left(A_{\mu}\right)$ is $2^{N_{0}}$. Hence there exists an element $x$ of $C_{\mu}-B_{\mu}$. Let $p_{\mu}$ 
be the first such element and $q_{\mu}=f_{\mu}\left(p_{\mu}\right)$. Denote by $A$ the set $E \cup\left\{p_{\xi} \mid \xi<\theta\right\}$.

The set $A$ is proved exact by an argument similar to that given in [2, pp. 325-326] and [3, Theorem 4]. In particular, the set $A$ is shown to be dense in $R$, and consequently any similarity transformation $f$ of $A$ into $A$ can be extended to become similarity transformation $f_{\nu}$ of $R$ into $R$. Therefore the point $f_{\nu}\left(p_{v}\right)$ is not in $A$, so that no such function $f$ can exist. Clearly each point of $A$ is a $c$-condensation point of $A$.

Let $p$ be an element of $R-A$. Then the set $B=\{p\} \cup A$ is exact, by Corollary 2 of Theorem 2 of [3], and has the property that $\bar{E}<\bar{B}<\lambda$. As $A$ is dense in $R$ and each point of $A$ is a $c$-condensation point of $A$, the set $B$ has the same property. Q.E.D.

The set $E$ for which $\bar{E} \equiv \lambda$, and those for which $\bar{E} \equiv \alpha \omega$, where $\alpha<\alpha 2$, have the following two properties:

$(\alpha) E$ has no fixed point;

$(\beta)$ problem $(\mathrm{P})$ always admits of a solution $\left({ }^{7}\right)$.

It is only natural, then, to inquire as to whether or not any set $E$ which satisfies $(\alpha)$ also satisfies $(\beta)$. In other words, suppose that $E$ is a simply ordered set which has no fixed points, and that $A$ is a subset of $E$ for which $\bar{A}<\bar{E}$. Does it follow that for some order type $\tau$ we have $\bar{A}<\tau<\bar{E}$ ? An elementary example shows that the answer to this question is in the negative. Specifically, let

$$
A=\left\{-\frac{n-1}{n} \mid 1<n<\omega\right\} \text { and } E=A \cup\left\{\frac{n-1}{n} \mid 1<n<\omega\right\} .
$$

Clearly there is no order type $\tau$ for which $\omega^{*}<\tau<\omega^{*}+\omega$.

That the power of $E-A$ in the above example cannot be lowered is contained in

THEOREM 2.5. If $E$ has no fixed points and $A$ is a subset of $E$ for which $\bar{A}<\bar{E}$, then the power of $E-A$ is $\geqq \aleph_{0}$.

Proof. From (I) of this section, we can conclude that

$\left(^{*}\right)$ if $G$ is a subset of $E$ for which $\bar{G} \equiv \bar{E}$, and if $x_{0}$ is a fixed point of $G$, then $x_{0}$ is also a fixed point of $E$.

Now assume that the power $E-A$ is $n+1$, where $n$ is a non-negative integer. Let $E-A=\left\{x_{0}, x_{1}, \cdots, x_{n}\right\}$. Since $x_{n}$ is not a fixed point of $E$, it follows that

$$
\bar{A}<\bar{E} \equiv \overline{A \cup\left\{x_{i} \mid i \leqq n-1\right\}} .
$$

From $\left({ }^{*}\right)$, we see that no point of the set $A \cup\left\{x_{i} \mid i \leqq n-1\right\}$ is fixed in $A$ $\cup\left\{x_{i} \mid i \leqq n-1\right\}$. Repeating the process $n-1$ times leads us to the result

(7) See Theorem 7 of [6], Theorem 1.3 and (III) of $\$ 2$. 
that $\bar{A}<\overline{A \cup\left\{x_{0}\right\}}$, and repeating once more, to $\bar{A}<\bar{A}$. From this contradiction, we arrive at the conclusion that the power of $E-A$ cannot be $n+1$, and thus must be $\geqq \boldsymbol{N}_{0}$.

3. Decompositions of sets. In this section we shall be concerned with the decomposition of linear sets into the union of disjoint sets which satisfy some special conditions.

Lemma 3.1. Let $E$ have property $\mathrm{A}^{\prime}$ and $F$ be a set of real numbers of power $<2^{\aleph_{0}}$. Then the set $G=E \cup F$ has property A.

Proof. Suppose that $A$ and $B$ are two similar, disjoint, subsets of $G$, of power $2^{\aleph_{0}}$ each. Since the power of $F$ is $<2^{\aleph_{0}}$, the power of $A \cap E$ is $2^{\aleph_{0}}$. Let $C$ be a subset of $B$ which is similar to $A \cap E$. It follows that the power of set $C \cap E$ is $2^{N_{0}}$. Let $D$ be a subset of $A \cap E$ which is similar to $C \cap E$. Then $D$ and $C \cap E$ are two disjoint similar subsets of $E$ of power $2^{N_{0}}$ each. As this contradicts $E$ having property A, $G$ must have property A.

THEOREM 3.1. Each linear set $E$ of power $2^{\aleph_{0}}$ is the union of $2^{\mathrm{N}_{0}}$ disjoint subsets $E_{\xi}$, where each $E_{\xi}$ is an exact set of power $2^{\mathbb{N o}_{0}}$, and has property A.

Proof. Let $E$ be the union of disjoint sets $D_{\xi}, \xi<\theta$, where the power of each set is $2^{\mathrm{N}_{0}}$. By Theorems 5 and 4 of [3], each set $D_{\xi}$ contains a subset $B_{\xi}$ which is exact and has property A. Let $C=\bigcup_{\xi<\theta}\left(D_{\xi}-B_{\xi}\right)$. Well order the elements of $C$ into the sequence $\left\{x_{\xi}\right\}, \xi<\mu<\theta$. Let $E_{\xi}=B_{\xi} \cup\left\{x_{\xi}\right\}$ for $\xi<\mu$, and $E_{\xi}=B_{\xi}$ for $\xi \geqq \mu$. By Lemma 3.1 above and Corollary 2 of Theorem 2 of [3] the sets $E_{\xi}$ satisfy the conclusion of the theorem. Q.E.D.

We shall now show that there exist two disjoint, exact subsets, $F$ and $G$, of the set $C=\{x \mid 0<x \leqq 1, x \in R\}$, whose set union is $C$. Let $H$ be the set of those similarity transformations of $C$ into $C$ which are not the identity. Well order the elements of $H$ and $C$ into the two sequences $\left\{f_{\xi}\right\}, \xi<\theta$, and $\left\{x_{\xi}\right\}$, $\xi<\theta$. Let $J_{\xi}=\left\{x \mid f_{\xi}(x) \neq x, x \in C\right\}$. Denote by $f_{\xi}^{*}$ the inverse function of $f_{\xi}$. Let $p_{0}^{1}=x_{0}$ and $q_{0}^{1}=x_{1}$. Let $M_{0}=\left\{p_{0}^{1}, q_{0}^{1}, f_{0}^{*}\left(p_{0}^{1}\right), f_{0}^{*}\left(q_{0}^{1}\right)\right\}$, $p_{0}^{2}$ be the first element in the set $J_{0}-M_{0}$, and $q_{0}^{2}=f_{0}\left(p_{0}^{2}\right)$. Let $q_{0}^{3}$ be the first element in the set $J_{0}-\left[M_{0} \cup\left\{p_{0}^{2}, q_{0}^{2}, f_{0}^{*}\left(p_{0}^{2}\right)\right\}\right]$ and $p_{0}^{3}=f_{0}\left(q_{0}^{3}\right)$. We continue by induction. Assume that the points $p_{\xi}^{i}$ and $q_{\xi}^{i}$ have been defined for $i=1,2,3$, and $\xi<\mu$. Let $p_{\mu}^{1}$ and $q_{\mu}^{1}$ be the first two elements in the set $C-N_{\mu}$, where $N_{\mu}=\left\{p_{\xi}^{i}, q_{\xi}^{i} \mid i\right.$ $=1,2,3 ; \xi<\mu\}$. Let $p_{\mu}^{2}$ be the first element in the set $J_{\mu}-P_{\mu}$, where

$$
P_{\mu}=N_{\mu} \cup f_{\mu}^{*}\left(N_{\mu}\right) \cup\left\{p_{\mu}^{1}, q_{\mu}^{1}, f_{\mu}^{*}\left(p_{\mu}^{1}\right), f_{\mu}^{*}\left(q_{\mu}^{1}\right)\right\},
$$

and $q_{\mu}^{2}=f_{\mu}\left(p_{\mu}^{2}\right)$. Let $q_{\mu}^{3}$ be the first element in the set $J_{\mu}-\left[P_{\mu} \cup\left\{p_{\mu}^{2}, q_{\mu}^{2}, f_{\mu}^{*}\left(p_{\mu}^{2}\right)\right\}\right]$ and $p_{\mu}^{3}=f_{\mu}\left(q_{\mu}^{3}\right)$. Let

$$
F=\left\{p_{\xi}^{i} \mid i=1,2,3 ; \xi<\theta\right\} \text { and } G=\left\{q_{\xi}^{i} \mid i=1,2,3 ; \xi<\theta\right\} .
$$

Clearly the sets $F$ and $G$ are disjoint and their set union is $C$. Furthermore, 
the sets $F$ and $G$ are each dense in $G$, so that each similarity transformation of $F$ and $G$ into $C$ can be extended to be a similarity transformation of $C$ into $C$. By using an argument similar to that in Theorem 2.4, the sets $F$ and $G$ are seen to be exact.

Using this result two disjoint, similar subsets, $A$ and $B$ of $R$ can be found for which $R=A \cup B$ and $\bar{A}<\bar{R}$. To see this let $f_{n}(x)=n+x$ for any integer $n$. Let $M$ denote the set which consists of zero and the positive and negative integers. Denote by $A$ the set

$$
\left[\bigcup_{n} f_{M} f_{2 n}(F)\right] \cup\left[\bigcup_{n} f_{M} f_{2 n+1}(G)\right]
$$

and by $B$ the set

$$
\left[\bigcup_{n} \in_{M} f_{2 n+1}(F)\right] \cup\left[\underset{n \in M}{\bigcup} f_{2 n}(G)\right] .
$$

Clearly the sets $A$ and $B$ have the desired properties. Notice that the set $A$ contains no fixed point. This is no accident which is due to the particular set $A$ constructed, as the following theorem shows.

THEOREM 3.2. Let $A$ and $B$ be any two similar, disjoint subsets of $R$ whose set union is $R$. Then $A$ (and thus $B$ ) cannot contain any fixed points.

Proof. Suppose that $A$ contains the fixed point $y_{0}$. This implies that $\bar{A}<\bar{R}$, so that the set $A$ cannot contain any interval of $R$. Thus the complement of $A$, in this case $B$, is a dense subset of $R$. Similarly, $A$ is a dense subset of $R$. Since $A$ and $B$ are similar, there is a similarity transformation $f$ of $A$ onto $B$. Let $g(x)$ be the function defined as follows: If $x$ is in $A$, then let $g(x)=f(x)$. If $x_{0}$ is in $B$, then let $g\left(x_{0}\right)=\lim _{x \rightarrow x_{0}-} g(x)$, where $x$ runs only through elements of $A$. Since $A$ is a dense subset of $R$, the element $g\left(x_{0}\right)$ is well defined. Suppose that for some $x_{0}$ in $B, g\left(x_{0}\right)$ is in $B$. Since $f$ maps $A$ onto $B$, an element $\bar{x}$ in $A$ can be found for which $g(\bar{x})=g\left(x_{0}\right)$. As $A$ is a dense subset of $R$ and $f$ is a similarity transformation, it follows that $\bar{x}=\lim _{x<x_{0}} x_{0}$. Consequently the point $\bar{x}$ and $x_{0}$ are the same, i.e., $\bar{x}=x_{0}$. However, this contradicts the fact that $x_{0}$ is an element of $B$. Thus, if $x_{0}$ is in $B$, then $g\left(x_{0}\right)$ is in $A$. Therefore $g$ is a similarity transformation of $R$ into $R$ for which $g(A) \subseteq B$ and $g(B) \subseteq A$. If $h$ is the function which is defined by $h(x)=g[g(x)]$ for $x$ in $A$, then $h$ is a similarity transformation of $A$ into $A$ such that $h\left(y_{0}\right) \neq y_{0}$. But this contradicts the assumption that $y_{0}$ is a fixed point of $A$. The contradiction establishes that $A$ contains no fixed point. Q.E.D.

Since two exact sets, $A$ and $B$, for which $\bar{A} \equiv \bar{B}$, are also similar we have

Corollary 3.1. Let $A$ and $B$ be two disjoint subsets of $R$, for which $\bar{A} \equiv \bar{B}$, and whose set union is $R$. Then $A$ cannot be exact.

The example stated prior to Theorem 3.2 is also useful as a counterexample. 
One might well ask if Theorem 1.2 can be generalized to obtain an order type $\tau<\bar{E}$ for which there is no order type $\mu$, of power $2^{\aleph_{0}}$, so that $\mu \leqq \sigma$ and $\mu \leqq \tau$. Let $A$ and $B$ be the sets described in the example, $f$ the similarity transformation which maps $A$ onto $B$, and $f^{*}$ the inverse function of $f$. Clearly $\sigma=\bar{A}<\lambda$. Let $\tau$ be any order type of power $2^{\aleph_{0}}$ which is $<\lambda$ and incomparable with $\sigma$. Let $D$ be any subset of $R$ for which $\bar{D}=\tau$, and let $H=B \cap D$. If the power of $H$ is $2 N_{0}$, then

has the property that

$$
\bar{H}=\overline{f^{*}(H)}
$$

$$
\bar{H} \leqq \bar{D} \text { and } \overline{f^{*}(H)} \leqq \bar{A} .
$$

If the power of $H$ is $\left\langle 2^{\aleph_{0}}\right.$, then the power of $G=A \cap D$ is $2^{\aleph_{0}}$. Furthermore, $\bar{G} \leqq \bar{A}$ and $\bar{G} \leqq \bar{D}$. In either of the cases then there exists an order type $\mu$, where $\mu \leqq \sigma$ and $\mu \leqq \tau$.

As the final result of this section we prove

THeOREM 3.3. If $f$ is a similarity transformation of $E$ into itself such that $f(x) \neq x$ for each $x$ in $E$, then $E$ can be decomposed into $\boldsymbol{\aleph}_{0}$ disjoint sets $E_{n}$, where $\bar{E}_{n} \equiv \bar{E}_{m}$.

Proof. Let $f^{1}(x)=f(x)$ and $f^{n}(x)=f\left[f^{n-1}(x)\right]$ for $n>1$. We define a relation $R$ on $E$ as follows:

For every pair of elements $x$ and $y$ of $E$, write $x R y$ if, for some positive integer $n$, at least one of the conditions (1) $x \leqq y$ and $f^{n}(x) \geqq y$; (2) $y \leqq x$ and $f^{n}(y) \geqq x$; (3) $y \leqq x$ and $f^{n}(x) \leqq y$; (4) $x \leqq y$ and $f^{n}(y) \leqq x$ is valid.

The relation $R$ is an equivalence relation on $E$, i.e., $(\alpha) x R x$ for each $x$ in $E,(\beta) x R y$ implies $y R x$, and $(\gamma) x R y$ and $y R z$ implies that $x R z$. Furthermore, suppose that $x R y$ and that $z$ is an element of $E$ for which $x<z<y$, or $y<z<x$. Then $z R x$ is also true. The aforementioned two properties of $R$ imply that the equivalence classes derived from the relation $R$ in the standard manner are also intervals of $E$. Therefore, to prove the conclusion of the theorem, it is sufficient to decompose each interval $D$ into $\boldsymbol{\aleph}_{0}$ disjoint sets $D_{n}$, where $\bar{D}_{n}$ $\equiv \bar{D}_{m}$ for $n \neq m$.

Let $x_{0}$ be an element of $D$ and $f\left(x_{0}\right)>x_{0}$. Consider the sets

$$
\begin{aligned}
A_{1} & =\left\{x \mid x_{0} \leqq x<f\left(x_{0}\right)\right\}, & & \\
A_{n} & =\left\{x \mid f^{n-1}\left(x_{0}\right) \leqq x<f^{n}\left(x_{0}\right)\right\} & & \text { for } n>1, \\
A_{-1} & =\left\{x \mid f(x) \in A_{1}\right\}, & & \\
A_{-n} & =\left\{x \mid f(x) \in A_{-n+1}\right\} & & \text { for } n>1 .
\end{aligned}
$$

It is to be understood that the sets $A_{-n}$ may be empty from some integer $\bar{n}$ on. Note that 


$$
D=\left[\underset{0<n<\omega}{\bigcup} A_{n}\right] \cup\left[\underset{0<n<\omega}{\bigcup} A_{-n}\right] .
$$

Arrange the set of positive integers into $\aleph_{0}$ disjoint sets $H_{n}, n \geqq 1$, each $H_{n}$ being enumerable. Let

$$
D_{n}=\left[\bigcup_{m} \in_{H_{n}} A_{m}\right] \cup\left[A_{-n}\right] .
$$

Consider the two sets $D_{j}$ and $D_{k}$. Since the order types of the two sets, $C_{j}$ $=\{-j\} \cup H_{j}$ and $C_{k}=\{-k\} \cup H_{k}$, are $\omega$, there exists a similarity transformation $h_{j k}$ which maps $C_{j}$ into $C_{k}$ such that $h_{j k}(r)>r$ for $r$ in $C_{j}$. Therefore for $m$ an integer in $C_{j}, A_{m}$ is similar to a subset of $A_{h_{j k}(m) \text {. Hence }} \bar{D}_{j} \leqq \bar{D}_{k}$. By symmetry it follows that $\bar{D}_{k} \leqq \bar{D}_{j}$. Consequently $\bar{D}_{k} \equiv \bar{D}_{j}$.

If $x_{0}$ is an element of $E$ and $f\left(x_{0}\right)<x_{0}$, then an analogous decomposition is possible. Q.E.D.

REMARK. Examples exist which show the fact that $\bar{E}_{n} \equiv \bar{E}$ cannot be added to the conclusion of Theorem 3.3. To see this let $A$ be any dense, exact subset of $(0,1)$ in which each point of $A$ is a $c$-condensation point of $A$. Furthermore, let $A$ have property A. Let $\left\{y_{n}\right\}, n<\omega$, be a decreasing sequence of points of $A$ whose limit is 0 . For each integer $n \geqq 1$ let $f_{n}$ and $g_{n}$ be similarity transformations of $A$ into $(-n-1,-n)$ and $(n, n+1)$ respectively. Denote by $A_{n}$ the set $A \cap\left\{x \mid x<y_{n-1}, x \in R\right\}$. Let $E=\left[\bigcup_{0<n<\omega} f_{n}\left(A_{n}\right)\right]$ $\cup\left[\mathrm{U}_{0<n<\omega} g_{n}(A)\right]$. We leave to the reader the proof that $\bar{E}_{n} \equiv \bar{E}$ is not possible.

The question arises as to conditions under which the hypotheses of Theorem 3.3 are satisfied. The following are stated without proof:

( $\alpha$ ) Let $A$ be a set of power $\geqq 2$ which has the property that for each element $x_{0}$ in $A, \bar{A} \equiv \overline{B\left(x_{0}\right)}$, where $B\left(x_{0}\right)=\left\{x \mid x \geqq x_{0}\right\}$. Then there exists a similarity transformation $f$ of $A$ into itself such that $f(x) \neq x$ for $x$ in $A$.

$(\beta)$ Let $B$ be a set of power $\geqq 2$ which has the property that for each $x_{0}$ in $B, \bar{B} \equiv \overline{B\left(x_{0}\right)}$, where $B\left(x_{0}\right)=\left\{x \mid x \leqq x_{0}\right\}$. Then there exists a similarity transformation $f$ of $B$ into itself such that $f(x) \neq x$ for $x$ in $B$.

$(\gamma)$ Let $E=A \cup B$, where $a<b$ for each $a$ in $A$ and each $b$ in $B$. If $A$ has the properties of the set $A$ in $(\alpha)$, and $B$ the properties of the set $B$ in $(\beta)$, then there exists a similarity transformation $f$ of $E$ into itself such that $f(x) \neq x$ for $x$ in $E$.

4. Two special sets. In this section we shall construct two sets which have special properties.

THEOREM 4.1. There exists a set $E$ which has the following four properties:

(1) $E$ is a dense, exact subset of $(0,1)$;

(2) each point of $E$ is a c-condensation point of $E$;

(3) $E$ contains a dense subset $A$ which has no fixed point;

(4) the power of $E-A$ is $\aleph_{0}$. 
Proof. Denote by $G_{0}$ any dense, exact subset of the open interval $(0,1)$, in which each point of $G_{0}$ is a $c$-condensation point of $G_{0}$. Furthermore, let $G_{0}$ have property A. For $n=1,2, \cdots$ let $f_{n}$ be a similarity transformation of $G_{0}$ onto a dense subset of the open interval $((n-1) / n, n /(n+1))$. Let $\left\{x_{n}\right\}$, $0<n<\omega$ be an increasing sequence of points of $G_{0}$ whose limit is the real number $1 / 2$. Denote by $G_{n}$ the set

$$
f_{n}\left[G_{0}-\left\{x_{i} \mid i<n\right\}\right],
$$

and by $E$ the set $\mathrm{U}_{0<n<\omega} G_{n}$. Each point of $E$ is a $c$-condensation point of $E$. Furthermore, $E$ is a dense subset of $(0,1)$. Now define $A$ as the set

$$
A=\underset{0<n<\omega}{\bigcup} f_{n}\left[G_{0}-\left\{x_{i} \mid i<\omega\right\}\right] .
$$

$A$ is a dense subset of $E$ and contains no fixed point. The power of $E-A$ is $\aleph_{0}$. The nontrivial part of the proof is in showing that $E$ is also exact.

To see this, suppose that $g$ is a similarity transformation of $E$ into $E$ which is not the identity, i.e., a point $y_{0}$ of $E$ can be found for which $g\left(y_{0}\right) \neq y_{0}$. All elements considered in the sequel are elements of $E$.

(a) First assume that $g\left(y_{0}\right)<y_{0}$. Let $k$ be the smallest integer for which there exists a point $z_{k}$ of $G_{k}$ satisfying the condition that $g\left(z_{k}\right)<z_{k}$. Since $g\left(y_{0}\right)<y_{0}$, the integer $k$ certainly exists. As $z_{k}$ is a $c$-condensation point of $E$, the power of the set

$$
H=\left\{x \mid g\left(z_{k}\right)<x \leqq z_{k}\right\}
$$

is $2^{N_{0}}$. It follows immediately that if $x$ is in $H$, then $g(x)<x$. Since

$$
g\left\{g\left[g\left(z_{k}\right)\right]\right\}<g\left[g\left(z_{k}\right)\right]<g\left(z_{k}\right),
$$

we conclude from the minimality property of $k$ that both $g\left(z_{k}\right)$ and $g\left[g\left(z_{k}\right)\right]$ are elements of $G_{k}$. Thus $H \cap g(H)=\phi, H \subseteq G_{k}$, and $g(H) \subseteq G_{k}$. These three conditions contradict the fact that $G_{0}$, and therefore also $G_{k}$, has property A.

(b) Suppose that $g\left(y_{0}\right)>y_{0}$. Then a set $G_{s}$ can be found so that for any element $x$ of $G_{s}, g(x)$ is in $G_{s+p(x)}$, where $p(x)>0$. For suppose that the element $y_{0}$ is in $G_{r}$ and that for some $y$, where $y_{0}<y \leqq g\left(y_{0}\right)$, the element $g(y)$ is also in $G_{r}$. Then $H_{1}=\left\{x \mid y_{0}<x<y\right\}$ and $H_{2}=g\left(H_{1}\right)$ are two similar disjoint subsets of $G_{r}$, of power $2^{\aleph_{0}}$ each. Since this contradicts $G_{r}$ having property A, the element $g(y)$ must be in $G_{r+p(y)}$, where $p(y)>0$. Denoting by $\bar{y}$ one such element $y$, let $s=r+p(\bar{y})$. Now suppose that $x$ is any element in $G_{s}$ and $x \leqq g(\bar{y})$. For $x^{*}<x$ and $x^{*}$ in $G_{s}$, consider $g(x)$. If $g(x)$ were to be in $G_{s}$, then the two disjoint subsets of $G_{s}, H_{3}=\left\{y \mid x^{*}<y<x\right\}$ and $g\left(H_{3}\right)$, would be similar, which would be a contradiction. Therefore $g(x)$ is in $G_{s+p(x)}$, where $p(x)>0$. For any element $x>g(\bar{y})$ in $G_{s}, g(x)>g[g(\bar{y})]$. Since $g[g(\bar{y})]$ is in $G_{s+p}[g(\bar{y})]$ $g(x)$ is in $G_{s+p(x)}$, where $p(x)>0$. Hence, for any element $x$ in $G_{s}, g(x)$ is in $G_{\imath+p(x)}$, where $p(x)>0$. 
Let $p$ and $q$ be any two positive integers, where $p<q$, and $z_{1}$ and $z^{2}$ any two elements of $G_{p}$ for which $z_{1}<f_{p}\left(x_{q-1}\right)<z_{2}$. Then there is no similarity transformation of $H_{4}=\left\{x \mid z_{1}<x<z_{2}\right\}$ into $G_{q}$. To see this suppose that $h$ is such a similarity transformation. We recall that $f_{q}\left(x_{q-1}\right)$ is not an element of $G_{q}$. Consider the point $h\left[f_{p}\left(x_{q-1}\right)\right]$. First suppose that $h\left[f_{p}\left(x_{q-1}\right)\right]<f_{q}\left(x_{q-1}\right)$. Designate by $f^{*}$ the inverse transformation of $f$. As the functions $h, f_{p}$, and $f_{q}$ are similarity transformations, the sets $f_{q}^{*}\left[h\left(H_{5}\right)\right]$ and $f_{p}^{*}\left(H_{5}\right)$ are similar, where

$$
H_{5}=\left\{x \mid z_{1}<x \leqq f_{p}\left(x_{q-1}\right)\right\} .
$$

Since $f_{p}^{*}\left(H_{5}\right)=\left\{y \mid f_{p}^{*}\left(z_{1}\right)<y \leqq x_{q-1}\right\} \quad$ and $f_{q}^{*}\left[h\left(H_{5}\right)\right] \subseteq\left\{y \mid f_{q}^{*}\left[h\left(z_{1}\right)\right]<y \leqq f_{q}^{*}\right.$ $\left.\cdot\left(h\left[f_{p}\left(x_{q-1}\right)\right]\right)<x_{q-1}\right\}$, it follows that the power of $f_{p}^{*}\left(H_{5}\right)-f_{q}^{*}\left[h\left(H_{5}\right)\right]$ is $2 \mathrm{~N}_{0}$. It is an immediate consequence of the set $G_{0}$ having property $\mathrm{A}$ that the sets $f_{p}^{*}\left(H_{5}\right)$ and $f_{q}^{*}\left[h\left(H_{5}\right)\right]$ cannot be similar. In an analogous manner, the assumption that $h\left[f_{p}\left(x_{q-1}\right)\right]>f_{q}\left(x_{q-1}\right)$ also leads to a contradiction. Consequently no such similarity transformation $h$ can exist.

Now consider the set $g\left(G_{s}\right)$, where for $x$ in $G_{s}, g(x)$ is an element of $G_{s+p(x)}$, $p(x)>0$. Suppose that $g\left(G_{s}\right) \subseteq \bigcup_{0<i \leqq n} G_{m_{i}}$, where

$$
s<m_{1}<m_{2}<\cdots<m_{n}
$$

are $n+1$ positive integers. By considering the location of the points $f_{8}\left(x_{m_{1}-1}\right)$, $f_{s}\left(x_{m_{2}-1}\right), \cdots, f_{s}\left(x_{m_{n}-1}\right)$ in conjunction with the result of the preceding paragraph, we see that "at best,"

$$
\begin{aligned}
& \left\{x \mid x \leqq f_{s}\left(x_{m_{1}-1}\right), x \in G_{s}\right\} \text { is mapped by } g \text { into } G_{m_{1}}, \\
& \left\{x \mid f_{s}\left(x_{m_{1}-1}\right)<x \leqq f_{s}\left(x_{m_{2}-1}\right)\right\} \text { is mapped by } g \text { into } G_{m_{2}}, \\
& \text {. . . . . . . . . . . . . . . . . . . . . . . } \\
& \left\{x \mid f_{s}\left(x_{m_{n-1}-1}\right)<x \leqq f_{s}\left(x_{m_{n}-1}\right)\right\} \text { is mapped by } g \text { into } G_{m_{n}} \text {. }
\end{aligned}
$$

Thus the set $\left\{x \mid f_{8}\left(x_{m_{n}-1}\right) \leqq x, x \in G_{s}\right\}$ is not mapped into $\cup_{0<i \leqq n} G_{m_{i}}$. In other words, for any integer $n_{0}$, there exists an element $x$ of $G_{s}$ and an integer $n_{1}>n_{0}$ so that $g(x)$ is in $G_{n_{1}}$. If $y$ is any element of $G_{s+1}$, then since $g$ is a similarity transformation, $g(y)>g(x)$ for any $x$ in $G_{s}$. But clearly this is impossible, since elements of $G_{s}$ are mapped into the sets $G_{m}$ for arbitrarily large $m$. Therefore the assumption that $g\left(y_{0}\right)>y_{0}$ leads to a contradiction.

Summarizing, we have seen that neither of the possibilities, $g\left(y_{0}\right)<y_{0}$ or $g\left(y_{0}\right)>y_{0}$, can occur for any point $y_{0}$ of $E$. Consequently there can be no similarity transformation of $E$ into $E$ except the identity, i.e., the set $E$ is exact.

THEOREM 4.2. There exists a set $E$ which has the following properties:

(1) $E$ is a linear set of power $2^{\mathrm{N}_{0}}$;

(2) E contains no fixed point;

(3) there exists no similarity transformation $f$ of $E$ into itself such that 
$f\left(x_{0}\right) \neq x_{0}$ for every element $x_{0}$ in $E$, i.e., for each similarity transformation $f$ of $E$ into itself, there exists a point $x_{0}$ so that $f\left(x_{0}\right)=x_{0}$.

(4) $E$ is not a closed set $\left.{ }^{8}\right)$.

Proof. Let $A$ be any dense, exact subset of $(0,1)$ in which each point of $A$ is a $c$-condensation point of $A$. Furthermore, let $A$ have property A. Let $\left\{y_{n}\right\}, n<\omega$, be a decreasing sequence of points of $A$ whose limit is 0 . For $n>0$ denote by $A_{n}$ the set

$$
A \cap\left\{x \mid y_{n}<x<y_{n-1}\right\}
$$

and by $f_{n}, n \geqq 1$, any similarity transformation of $A$ into $(n, n+1)$. Arrange the sequence of set $\left\{A_{n}\right\}$ into the sequence

$$
A_{1}, A_{2}, A_{1}, A_{2}, A_{3}, A_{1}, A_{2}, A_{3}, A_{4}, A_{1}, A_{2}, \cdots
$$

and let $B_{n}$ be the $n$th set of the sequence. Denote by $E$ the set

$$
\left[\underset{0<n<\omega}{\bigcup} A_{n}\right] \cup\left[\underset{0<n<\omega}{\bigcup} f_{n}\left(B_{n}\right)\right] .
$$

The details involved in showing that the set $E$ possesses the four enumerated properties are straightforward, and consequently are omitted.

\section{BibLIOGRAPHY}

1. G. Birkhoff, Lattice theory, Amer. Math. Soc. Colloquium Publications, vol. 25, rev. ed., New York, 1948.

2. B. Dushnik and E. W. Miller, Concerning similarity transformations of linearly ordered sets, Bull. Amer. Math. Soc. vol. 46 (1940) pp. 322-326.

3. S. Ginsburg, Order types and similarity transformations, submitted to the editors of Fund. Math.

4. J. C. Shepherdson, Well-ordered subseries of general series, Proc. London Math. Soc. (3) vol. 1 (1951) pp. 291-307.

5. W. Sierpinski, Remarques concernant les types de dimensions, Fund. Math. vol. 14 (1929) pp. 122-126. 264.

6. —_ Sur les types d'ordre des ensembles linéaires, Fund. Math. vol. 37 (1950) pp. 253-

UNIVERSITY OF MichigAN,

ANN Arbor, Mich.

UNIVERSITY OF MiAMI,

Coral Gables, Fla.

(8) See p. 41 , Ex. 6 of [1]. 